\title{
Gauge Coupling Unification
}

via

\section{A Novel Technicolor Model}

\author{
Sven Bjarke Gudnason* and Thomas A. Rytrovit \\ The Niels Bohr Institute, DK-2100 Copenhagen Ø, Denmark \\ Francesco SANNINd \\ University of Southern Denmark, DK-5230 Odense, and \\ The Niels Bohr Institute, DK-2100 Copenhagen Ø, Denmark
}

\begin{abstract}
We show that the recently proposed minimal walking technicolor theory together with a small modification of the Standard Model fermionic matter content leads to an excellent degree of unification of the gauge couplings. We compare the degree of unification with various time-honored technicolor models and the minimal supersymmetric extension of the Standard Model. We find that, at the one-loop level, the new theory provides a degree of unification higher than any of the other extensions above. The phenomenology of the present model is very rich with various potential dark matter candidates.
\end{abstract}

*Electronic address: gudnason@nbi.dk

†Electronic address: ryttov@nbi.dk

${ }^{\ddagger}$ Electronic address: sannino@nbi.dk 


\section{INTRODUCTION}

Coupling unification of all forces is a milestone for any extension of the standard model. In this letter we explore the unification issue for four dimensional extensions of the standard model (SM) in which the Higgs sector is replaced by a technicolor-like mechanism [1].

Much progress has been made recently in developing new models of technicolor type able to address the old problems for technicolor [2, 3, 44, 5, 6]. We have even identified and carefully studied within these models possible dark matter particles in the form of technibaryons [7]. Our technicolor differs from the more traditional models of technicolor used in the past since the technifermions transform according to higher dimensional representations of the underlying technicolor gauge group. This does not exclude the possibility that such fermions can be interpreted at some higher energy as transforming according to the fundamental representation of a higher rank group. One of the key features of these technicolor theories is that they walk [8, 9, 10, 11, 12, 13] for a very low number of technifermions and technicolors. Some of the problems of the simplest technicolor models, such as a large contribution to the oblique parameters [14], are alleviated [15, 16, 17] when considering new gauge dynamics in which the coupling does not run with respect to the energy scale but rather walks, i.e. evolves very slowly [8, 9, 10, 11, 12]. The simplest of such models which passes the electroweak precision tests, requires the technicolor matter to transform according to the two index representation [39] of the technicolor gauge group [2, 3, 4, 5]. In [18], the reader will find an exhaustive analysis of asymptotically free non-supersymmetric gauge theories with fermions in a given arbitrary higher dimensional representation of the $S U(N)$ gauge group and their use to dynamically break the electroweak symmetry.

The minimal walking technicolor (MWT) model has been introduced in [4, 5, 7] and consists of a two technicolor gauge theory with technifermions in the two-index symmetric (i.e. adjoint) representation of the technicolor gauge group. To avoid Witten's global $S U(2)$ anomaly [19], one introduces a new lepton family.

We start by investigating the one-loop evolution of the SM couplings once the SM Higgs is replaced by the MWT model. Quite surprisingly we find that the SM coupling constants unify much better than with the standard model Higgs being present. We compare our results with different time-honored technicolor models and show that either they are not competitive unification wise or they are not a prime candidate for walking technicolor the- 
ories according to recent results [18]. With a small modification of the technicolor gauge interactions we show how we can envision unification also with the technicolor coupling constant at the same energy scale.

Technicolor requires some other mechanism to provide the standard model fermion masses. This mechanism could have an effect on our results. We have estimated these corrections by providing a simple/minimal model which consists in adding a new Higgs field on the top of the minimal walking theory whose main purpose is to provide mass to standard model fermions. This construction has already been used in the literature [20, 21, 22, 23, 24, 25]. In a more natural theory this field will be replaced, perhaps, by some new strong dynamics. The model parametrizes our ignorance of a more fundamental extended technicolor theory. Surprisingly we find a degree of unification higher than in the theory without a mechanism for fermion mass generation. This result is very encouraging.

A general feature of a unified theory of the SM interactions is the prediction of the proton decay. A unification energy scale of the order of, or larger than, $10^{15} \mathrm{GeV}$ leads, typically, to phenomenologically acceptable proton decay rates. Despite the good, but yet not perfect, degree of unification - when compared, for example, to the minimal supersymmetric standard model result for unification - we discover that the proton decays too fast since the unification scale is quite low. To cure the proton decay problem we then add a QCD colored Weyl fermion transforming according to the adjoint representation of $S U(3)$ and one Weyl fermion transforming according to the adjoint of $S U_{L}(2)$. These fermions are known in supersymmetric extensions of the standard model as the gluino and the wino. We then compare with the supersymmetric predictions for unification at the same order in perturbation theory and discover that the present theory unifies better. Since we are not insisting on supersymmetry, there is no reason to expect the introduced fermions to be degenerate with the associated gauge bosons. Unification and naturality also suggest the presence of a Weyl fermion associated to the hypercharge gauge interaction and hence this degree of freedom is added in the model (the bino).

The reader may consider adding matter transforming according to even higher dimensional representations than the adjoint one or more generally higher than the two-index type matter. We know [18], however, that a very limited number of theories with fermions in higher dimensional representations remain asymptotically free when the rank of the gauge group increases. The unified gauge group must necessarily have quite a large rank con- 
straining matter to have at most two indices to insure asymptotic freedom. Recall, that to avoid low energy fine tuning of the coupling constants the unified gauge theory must be asymptotically free. This requirement, de facto, limits the maximum allowed representation in the theory.

The phenomenology, both for collider experiments and cosmology, of this novel extension of the standard model is very rich with many features common to both supersymmetry and technicolor.

\section{NOTATION AND CONVENTIONS}

The evolution of the coupling constant $\alpha_{n}$, at the one-loop level, of a gauge theory is controlled by

$$
\alpha_{n}^{-1}(\mu)=\alpha_{n}^{-1}\left(M_{Z}\right)-\frac{b_{n}}{2 \pi} \ln \left(\frac{\mu}{M_{Z}}\right),
$$

where $n$ refers to the gauge group being $S U(n)$, for $n \geq 2$ or $U(1)$, for $n=1$.

The first coefficient of the beta function $b_{n}$ is

$$
b_{n}=\frac{2}{3} T(R) N_{w f}+\frac{1}{3} T\left(R^{\prime}\right) N_{c b}-\frac{11}{3} C_{2}(G)
$$

where $T(R)$ is the Casimir[40] of the representation $R$ to which the fermions belong, $T\left(R^{\prime}\right)$ is the Casimir of the representation $R^{\prime}$ to which the bosons belong. $N_{w f}$ and $N_{c b}$ are respectively the number of Weyl fermions and the number of complex scalar bosons. $C_{2}(G)$ is the quadratic Casimir of the adjoint representation of the gauge group.

The SM gauge group is $S U(3) \times S U(2) \times U(1)$. We have three associated coupling constants which one can imagine to unify at some very high energy scale $M_{G U T}$. This means that the three couplings are all equal at the scale $M_{G U T}$, i.e. $\alpha_{3}\left(M_{G U T}\right)=\alpha_{2}\left(M_{G U T}\right)=$

$\alpha_{1}\left(M_{G U T}\right)$ with $\alpha_{1}=\alpha /\left(c^{2} \cos ^{2} \theta_{w}\right)$ and $\alpha_{2}=\alpha / \sin ^{2} \theta_{w}$, where $c$ is a normalization constant to be determined shortly.

Assuming one-loop unification using Eq. (1) for $n=1,2,3$, one finds the following relation

$$
\frac{b_{3}-b_{2}}{b_{2}-b_{1}}=\frac{\alpha_{3}^{-1}-\alpha^{-1} \sin ^{2} \theta_{w}}{\left(1+c^{2}\right) \alpha^{-1} \sin ^{2} \theta_{w}-c^{2} \alpha^{-1}} .
$$

In the above expressions the Weinberg angle $\theta_{w}$, the electromagnetic coupling constant $\alpha$ and the strong coupling constant $\alpha_{3}$ are all evaluated at the $Z$ mass. For a given particle content we shall denote the LHS of Eq. (3) by $B_{\text {theory }}$ and the RHS by $B_{\text {exp }}$. Whether 
$B_{\text {theory }}$ and $B_{\exp }$ agree is a simple way to check if the coupling constants unify. We shall use the experimental values $\sin ^{2} \theta_{w}\left(M_{Z}\right)=0.23150 \pm 0.00016, \alpha^{-1}\left(M_{Z}\right)=128.936 \pm 0.0049$, $\alpha_{3}\left(M_{Z}\right)=0.119 \pm 0.003$ and $M_{Z}=91.1876(21) \mathrm{GeV}$ [26]. The unification scale is given by the expression

$$
M_{G U T}=M_{Z} \exp \left[2 \pi \frac{\alpha_{2}^{-1}\left(M_{Z}\right)-\alpha_{1}^{-1}\left(M_{Z}\right)}{b_{2}-b_{1}}\right] .
$$

While the normalizations of the coupling constants of the two non-Abelian gauge groups are fixed by the appropriately normalized generators of the gauge groups, the normalization of the Abelian coupling constant is a priori arbitrary. The normalization of the Abelian coupling constant can be fixed by a rescaling of the hypercharge $Y \rightarrow c Y$ along with $g \rightarrow$ $g / c$. The normalization constant $c$ is chosen by imposing that all three coupling constants have a common normalization

$$
\operatorname{Tr}\left(c^{2} Y^{2}\right)=\operatorname{Tr}\left(T_{3}^{2}\right)
$$

where $T_{3}$ is the generator of the weak isospin and the trace is over all the relevant fermionic particles on which the generators act. It is sufficient to fix it for a given fermion generation (in a complete multiplet of the unification group).

The previous normalization is consistent with an $S U(5)$-type normalization for the generators of $U(1)$ of hypercharge, $S U(2)_{L}$ and $S U(3)_{c}$.

As well explained in the paper by $\mathrm{Li}$ and $\mathrm{Wu}[27]$ : At one-loop a contribution to $b_{3}-b_{2}$ or $b_{2}-b_{1}$ emerges only from particles not forming complete representations [41] of the unified gauge group. For example the gluons, the weak gauge bosons and the Higgs particle of the SM do not form complete representations of $S U(5)$ but ordinary quarks and leptons do. Here we mean that these particles form complete representations of $S U(5)$, all the way from the unification scale down to the electroweak scale. The particles not forming complete representations will presumably join at the unification scale with new particles and together then form complete representations of the unified gauge group. Note, that although there is no contribution to the unification point of the particles forming complete representations, the running of each coupling constant is affected by all of the particles present at low energy.

As a warm up, we consider the SM with $N_{g}$ generations. In this case we find $c=\sqrt{3 / 5}$, which is the same value one finds when the hypercharge is upgraded to one of the generators of $S U(5)$, and therefore the beta function coefficients are 


$$
\begin{aligned}
& b_{3}=\frac{4}{3} N_{g}-11, \\
& b_{2}=\frac{4}{3} N_{g}-\frac{22}{3}+\underbrace{\frac{1}{6}}_{\text {Higgs }}, \\
& b_{1}=\frac{3}{5}\left(\frac{20}{9} N_{g}+\frac{1}{6}\right)=\frac{4}{3} N_{g}+\underbrace{\frac{1}{10}}_{\text {Higgs }} .
\end{aligned}
$$

Here $N_{g}$ is the number of generations. It is clear that the SM does not unify since $B_{\text {theory }} \sim 0.53$ while $B_{\text {exp }} \sim 0.72$.

Note that the spectrum relevant for computing $B_{\text {theory }}$ is constituted by the gauge bosons and the standard model Higgs. The contribution of quarks and leptons drops out in agreement with the fact that they form complete representations of the unifying gauge group which, given the present normalization for $c$, is at least $S U(5)$. Hence the predicted value of $B_{\text {theory }}$ is independent of the number of generations. However the overall running for the three couplings is dependent on the number of generations and in Fig. 1 we show the behavior of the three couplings with $N_{g}=3$.

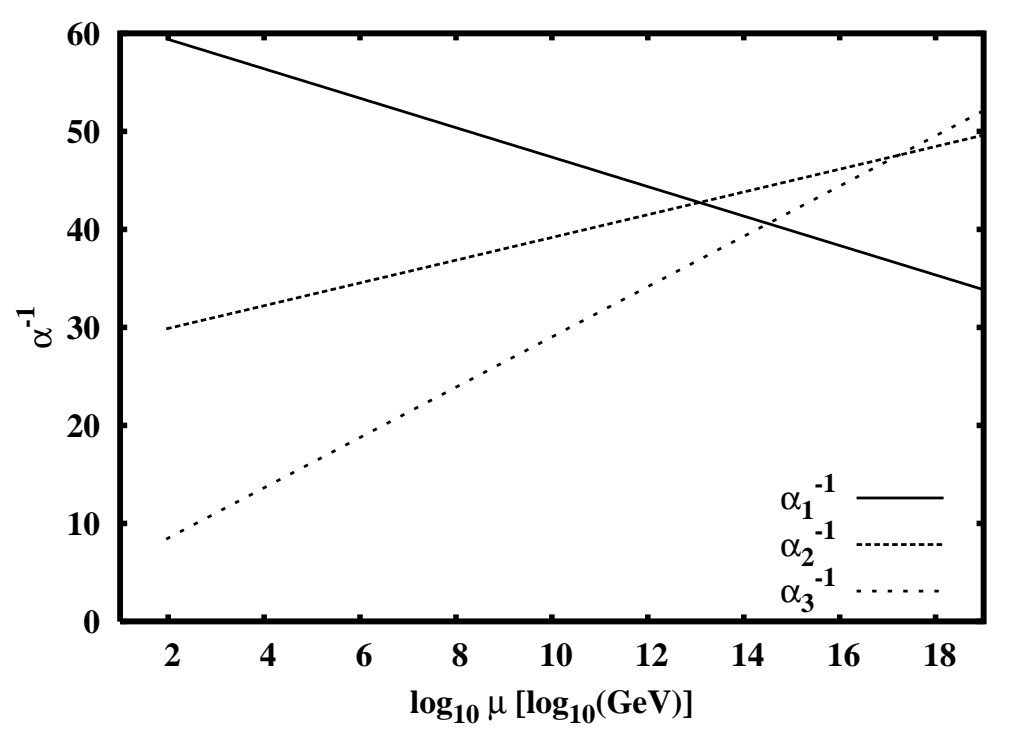

FIG. 1: The running of the three standard model gauge couplings. 


\section{STUDYING SU(3) $\times \mathrm{SU}(2) \times \mathrm{U}(1)$ UNIFICATION IN TECHNICOLOR}

Here we compare a few examples in which the standard model Higgs is replaced by a technicolor-like theory. A similar analysis was performed in [28]. In this section we press on recent phenomenological successful technicolor models with technimatter in higher dimensional representations and demonstrate that the simplest model helps unifying the SM couplings while other more traditional approaches are less successful. We also show that by a small modification of the technicolor dynamics, all of the four couplings can unify [42].

\section{A. Minimal Walking Technicolor (MWT)}

We examine what happens to the running of the SM couplings when the Higgs sector is replaced by the MWT theory proposed and investigated in [4, 5]. This model has technicolor group $S U(2)$ with two techniflavors in the two-index symmetric representation of the technicolor group. As already mentioned to avoid Witten's $S U(2)$ anomaly, the minimal solution is to add a new lepton family. Gauge anomaly cancellation as well as the previously mentioned anomaly do not uniquely fix the hypercharge assignment of the theory. Here we start by taking the simplest one in which the new techniparticles have electric $Q$ and hypercharge $Y$ mimicking the ones of the ordinary quarks and leptons

$$
\begin{array}{rlrl}
T_{L}^{(Q, Y)} & =\left(\begin{array}{c}
U_{L}^{\frac{2}{3}, \frac{1}{6}} \\
D_{L}^{-\frac{1}{3}, \frac{1}{6}}
\end{array}\right), & T_{R}^{(Q, Y)}=\left(U_{R}^{\frac{2}{3}, \frac{2}{3}}, D_{R}^{-\frac{1}{3},-\frac{1}{3}}\right), \\
\mathcal{L}_{L}^{(Q, Y)}=\left(\begin{array}{c}
\nu_{L, \zeta}^{0,-\frac{1}{2}} \\
\zeta_{L}^{-1,-\frac{1}{2}}
\end{array}\right), & \mathcal{L}_{R}^{(Q, Y)}=\left(\nu_{R, \zeta}^{0,0}, \zeta_{R}^{-1,-1}\right) .
\end{array}
$$

We still assume an $S U(5)$-type unification leading to $c^{2}=3 / 5$. The beta function coefficients will be those of the SM minus the Higgs plus the extra contributions from the techniparticles, ergo

$$
\begin{aligned}
& b_{3}=\frac{4}{3} N_{g}-11 \\
& b_{2}=\frac{4}{3} N_{g}-\frac{22}{3}+\frac{2}{3} \frac{1}{2}\left(\frac{2(2+1)}{2}+1\right)=\frac{4}{3}\left(N_{g}+1\right)-\frac{22}{3}, \\
& b_{1}=\frac{3}{5}\left(\frac{20}{9} N_{g}+\frac{20}{9}\right)=\frac{4}{3}\left(N_{g}+1\right),
\end{aligned}
$$

where $N_{g}$ is the number of ordinary SM generations. From this we see that $B_{\text {theory }}=0.68$ and $B_{\exp }=0.72$ and hence argue that we have a better unification than in the standard 


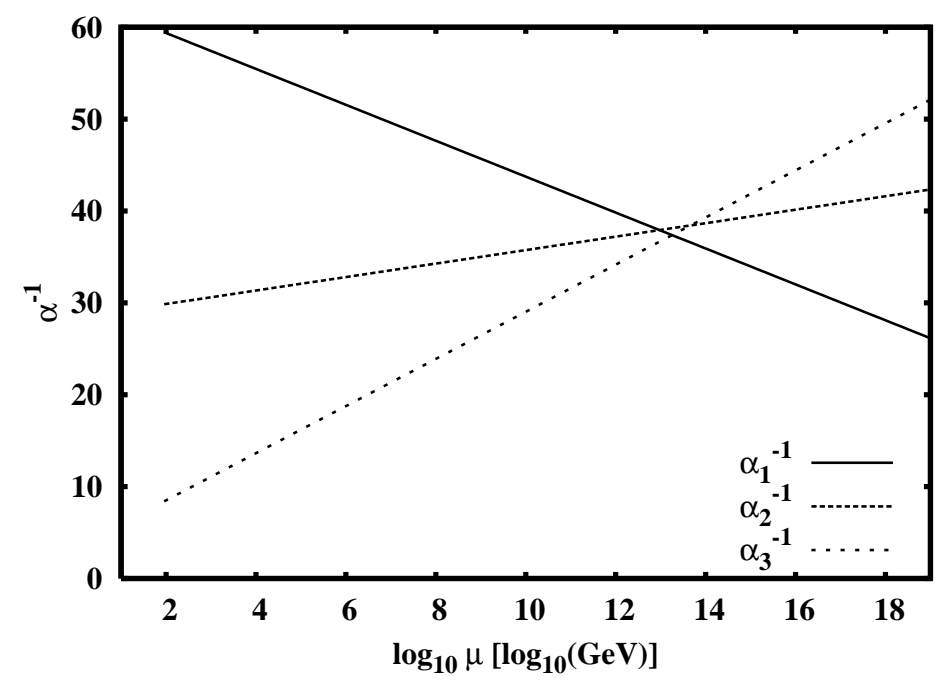

FIG. 2: The running of the SM gauge couplings in the presence of adjoint technifermions (the technicolor coupling is not included here).

model with an elementary Higgs. The running of the SM couplings is shown in Fig. 2 for three ordinary standard model generations. Note that the increase in $B_{\text {theory }}$ with respect to the SM is due to the fact that, typically, bosonic contributions are numerically suppressed with respect to fermionic ones and that, while $b_{1}-b_{2}=22 / 3$ receives only a contribution from the gauge sector, $b_{2}-b_{3}=11 / 3+4 / 3$ has two contributions, a gauge one and a fermionic one. These results are a direct consequence of the fact that we have no ordinary quarks related to the new leptonic family.

\section{B. Traditional Walking and Non-Walking One Family Model}

Here the technicolor particles also carry ordinary color and the technifermions constitute complete representations of $S U(5)$, hence the SM coupling unification receives no improvement with respect to the SM case. This is so since the numerical effect of the Higgs on the unification is small. For a one-family $S U(N)$ theory, we have $B_{\text {theory }}=1 / 2$.

\section{Partially Electroweak-Gauged Technicolor}

This approach consists in letting only one doublet of techniquarks transform non-trivially under the electroweak symmetries with the rest of the matter remaining in electroweak sin- 
glets, as suggested in [5] and later on used in [29]. In this case all techniquarks transform still under the technicolor gauge group and hence contribute to rendering the technicolor dynamics quasiconformal without affecting the leading perturbative contribution to the electroweak precision parameters. The reader can find a rather exhaustive investigation of walking technicolor theories with fermions in different representations of the technicolor gauge group in [18].

Denoting by $\operatorname{dim}\left[R_{T}\right]$ the dimension of the technicolor representation under which all the techniquarks transform we find the following result for $B_{\text {theory }}$

$$
B_{\text {theory }}=\frac{1}{2}\left[\frac{11+\operatorname{dim}\left[R_{T}\right]}{11-\operatorname{dim}\left[R_{T}\right] / 5}\right] .
$$

Since we have gauged only one technidoublet with respect to the weak interactions to avoid Witten's global anomaly with respect to the weak interactions we take $\operatorname{dim}\left[R_{T}\right]$ to be even. We have used the following hypercharge assignment free from gauge anomalies

$$
Y\left(T_{L}^{a}\right)=0, \quad Y\left(U_{R}^{a}, D_{R}^{a}\right)=\left(\frac{1}{2},-\frac{1}{2}\right), \quad a=1, \ldots, \operatorname{dim}\left[R_{T}\right]
$$

for the technidoublet charged under the electroweak interactions.

We find that $B_{\text {theory }}$ is 0.73 for $\operatorname{dim}\left[R_{T}\right]=4$ and it increases with larger values of $\operatorname{dim}\left[R_{T}\right]$. We can also consider the case $\operatorname{dim}\left[R_{T}\right]$ odd while solving Witten's anomaly by adding for example a new weak doublet uncharged under technicolor. We see that from the unification point of view partially electroweak gauged technicolor models are comparable with the MWT model presented earlier.

However, for the model to be phenomenologically viable the new technicolor theory should pass the electroweak precision constraints. A complete list of walking-type technicolor theories passing the precision tests can be found in [18]. The simplest unification condition requires the technicolor representation, in this case, to be four dimensional. This can only be achieved when the technifermions are arranged in the fundamental representation of the $S U$ (4)-technicolor gauge group. According to Table III in [18] one needs, at least, fifteen techniflavors for the theory to have a walking behavior with a reasonable $S$ parameter. In [18], this theory has not been listed as a prime candidate and hence will not be considered further here. 


\section{The Technicolor Coupling Constant}

Until now we have not discussed the technicolor coupling constant $\alpha_{T C}$. It is possible that the technicolor interaction does not unify with the other three forces or unifies later. A single step unification is though esthetically more appealing to us. Here we focus on the minimal walking theory which has already shown to be a promising theory for the unification of the standard model couplings. Remembering that the Casimir of the two-index symmetric representation of $S U\left(N_{T C}\right)$ is $\left(N_{T C}+2\right) / 2$ the first coefficient of the beta function $b_{T C}$ is easily found to be

$$
b_{T C}=\frac{2}{3}\left(N_{T C}+2\right) N_{f}-\frac{11}{3} N_{T C},
$$

where $N_{T C}$ is the number of technicolors and $N_{f}$ is the number of techniflavors. For two colors and two flavors we find $b_{T C}=-2$. Observing that, somewhat accidentally, also $b_{2}=-2$ for three ordinary SM generations, we conclude that the technicolor coupling constant cannot unify with the other three couplings at the same point. We are assuming, quite naturally, that the low energy starting points of $\alpha_{2}$ and $\alpha_{T C}$ are different.

Insisting that the technicolor coupling constant must unify with the other coupling constants at $M_{G U T}$, we need to modify at a given scale $X<M_{G U T}$ either the overall running of the SM couplings or the one of technicolor. To make less steep the running of the SM couplings one could add new generations. To avoid the loss of asymptotic freedom for the week coupling we find that at most only one entire new SM like generation can be added at an intermediate scale. If we, however, choose not to modify the running of the SM coupling constants, the running of the technicolor coupling constant must at some point $X<M_{G U T}$ become steeper. This can be achieved by enhancing the number of technigluons and lowering the contribution due to the techniquarks at the scale $X$. An elegant way to implement this idea is to imagine that the techniquarks - belonging to the three dimensional two-index symmetric representation of $S U(2)$ - are embedded in the fundamental representation of $S U(3)$ at the scale $X$. At energies below $X$ we have $b_{T C}^{<X}=-2$ and for energies larger than $X$ we have $b_{T C}^{>X}=-29 / 3$. If we take the technicolor coupling to start running at the electroweak scale $M_{E W} \sim 246 \mathrm{GeV}$ and unifying with the three SM couplings at the unification scale we find an expression for the intermediate scale $X$

$$
\ln X=\frac{1}{b_{T C}^{<X}-b_{T C}^{>X}}\left\{2 \pi\left(\alpha_{T C}^{-1}\left(M_{E W}\right)-\alpha_{T C}^{-1}\left(M_{G U T}\right)\right)+b_{T C}^{<X} \ln M_{E W}-b_{T C}^{>X} \ln M_{G U T}\right\} .
$$


If we take the starting point of the running of the technicolor coupling to be the critical coupling close to the conformal window we have $\alpha_{T C}\left(M_{E W}\right)=\pi /\left(3 C_{2}(\square \square)\right)=\pi / 6$. Also using the numbers $\alpha_{T C}\left(M_{G U T}\right)=\alpha_{i}\left(M_{G U T}\right) \sim 0.026, i=1,2,3, M_{G U T} \sim 9.45 \times 10^{12} \mathrm{GeV}$ we find the intermediate scale to be $X \sim 830 \mathrm{GeV}$.

\section{E. Proton Decay}

Grand Unified Theories lead, generally, to proton decay. Gauge bosons of mass $M_{V}<M_{G U T}$ are responsible for the decay of the proton into $\pi^{0}$ and $e^{+}$. The lifetime of the proton is estimated to be [31]

$$
\begin{aligned}
\tau & =\frac{4 f_{\pi}^{2} M_{V}^{4}}{\pi m_{p} \alpha_{G U T}^{2}(1+D+F)^{2} \alpha_{N}^{2}\left[A_{R}^{2}+\left(1+\left|V_{u d}\right|^{2}\right)^{2} A_{L}^{2}\right]} \\
& =\left(\frac{M_{G U T}}{10^{16} \mathrm{GeV}}\right)^{4}\left(\frac{\alpha_{G U T}^{-1}}{35}\right)^{2}\left(\frac{0.015 \mathrm{GeV}^{3}}{\alpha_{N}}\right)^{2}\left(\frac{2}{\mathcal{A}}\right)^{2} 2.7 \times 10^{35} \mathrm{yr},
\end{aligned}
$$

where we have used $f_{\pi}=0.131 \mathrm{GeV}$, the chiral Lagrangian factor $1+D+F=2.25$, the operator renormalization factors $\mathcal{A} \equiv A_{L}=A_{R}$ and the hadronic matrix element is taken

from lattice results [32] to be $\alpha_{N}=-0.015 \mathrm{GeV}^{3}$. Following Ross [33], we have estimated $\mathcal{A} \sim 2$ but a larger value $\sim 5$ is quoted in [31] . The lower bound on the unification scale comes from the Super-Kamiokande limit $\tau>5.3 \times 10^{33}$ yr [34]

$$
M_{G U T}>M_{V}>\left(\frac{35}{\alpha_{G U T}^{-1}}\right)^{1 / 2}\left(\frac{\alpha_{N}}{-0.015 \mathrm{GeV}^{3}}\right)^{1 / 2}\left(\frac{\mathcal{A}}{2}\right)^{1 / 2} 3.7 \times 10^{15} \mathrm{GeV}
$$

In the MWT model extension of the SM we find $\alpha_{G U T}^{-1} \sim 37.5$ and $M_{G U T} \sim 10^{13} \mathrm{GeV}$ yielding too fast proton decay.

\section{F. Constructing a Simple Unifying Group}

We provide a simple embedding of our matter content into a unifying gauge group. To construct this group we first summarize the charge assignments in table \. For simplicity we have considered right transforming leptons only for the charged ones. Also, the techniquarks are classified as being fundamentals of $S O(3)$ rather than adjoint of $S U(2)$. Except for topological differences, linked to the center group of the two groups, there is no other difference. This choice allows us to show the resemblance of the technicolor fermions with 
TABLE I: Quantum Numbers of the MWT + One SM Family

\begin{tabular}{c|c|c|c|c} 
& $S O_{T C}(3)$ & $S U_{c}(3)$ & $S U_{L}(2)$ & $U_{Y}(1)$ \\
\hline \hline$q_{L}$ & 1 & 3 & 2 & $1 / 6$ \\
$u_{R}$ & 1 & 3 & 1 & $2 / 3$ \\
$d_{R}$ & 1 & 3 & 1 & $-1 / 3$ \\
$L$ & 1 & 1 & 2 & $-1 / 2$ \\
$e_{R}$ & 1 & 1 & 1 & -1 \\
\hline$T_{L}$ & 3 & 1 & 2 & $1 / 6$ \\
$U_{R}$ & 3 & 1 & 1 & $2 / 3$ \\
$D_{R}$ & 3 & 1 & 1 & $-1 / 3$ \\
$\mathcal{L}_{L}$ & 1 & 1 & 2 & $-1 / 2$ \\
$\zeta_{R}$ & 1 & 1 & 1 & -1
\end{tabular}

ordinary quarks. We can now immediately arrange each SM family within an ordinary $S U(5)$ gauge theory. The relevant question is how to incorporate the technicolor sector (here we mean also the new Lepton family). An easy way out is to double the weak and hypercharge gauge groups as described in table II. This assignment allows us to arrange the low energy matter fields into complete representations of $S U(5) \times S U(5)$. To recover the low energy assignment one invokes a spontaneous breaking of the group down to $S O(3)_{T C} \times$ $S U_{c}(3) \times S U_{L}(2) \times U_{Y}(1)$ [43]. We summarize in table II] the technicolor and SM fermions transformation properties with respect to the grand unified group. Here the fields $A$ and $F$ are standard Weyl fermions and the gauge couplings of the two $S U(5)$ groups need to be the same. We have shown here that it is easy to accommodate all of the matter fields in a single semi-simple gauge group. This is a minimal embedding and others can be envisioned. New fields must be present at the grand unified scale (and hence will not affect the running at low energy) guaranteeing the desired symmetry breaking pattern. 
TABLE II: MWT + One SM Family enlarged gauge group

\begin{tabular}{c|c|c|c|c|c|c} 
& $S O_{T C}(3)$ & $S U_{1}(2)$ & $U_{1}(1)$ & $S U_{c}(3)$ & $S U_{2}(2)$ & $U_{2}(1)$ \\
\hline \hline$q_{L}$ & 1 & 1 & 0 & 3 & 2 & $1 / 6$ \\
$u_{R}$ & 1 & 1 & 0 & 3 & 1 & $2 / 3$ \\
$d_{R}$ & 1 & 1 & 0 & 3 & 1 & $-1 / 3$ \\
$L$ & 1 & 1 & 0 & 1 & 2 & $-1 / 2$ \\
$e_{R}$ & 1 & 1 & 0 & 1 & 1 & -1 \\
\hline$T_{L}$ & 3 & 2 & $1 / 6$ & 1 & 1 & 0 \\
$U_{R}$ & 3 & 1 & $2 / 3$ & 1 & 1 & 0 \\
$D_{R}$ & 3 & 1 & $-1 / 3$ & 1 & 1 & 0 \\
$\mathcal{L}_{L}$ & 1 & 2 & $-1 / 2$ & 1 & 1 & 0 \\
$\zeta_{R}$ & 1 & 1 & -1 & 1 & 1 & 0
\end{tabular}

TABLE III: GUT

\begin{tabular}{c|c|c} 
& $S U(5)$ & $S U(5)$ \\
\hline \hline $\bar{A}_{S M}$ & 1 & $\overline{10}$ \\
$F_{S M}$ & 1 & 5 \\
\hline $\bar{A}_{M W T}$ & $\overline{10}$ & 1 \\
$F_{M W T}$ & 5 & 1
\end{tabular}

\section{G. Providing Mass to the fermions}

We have not yet considered the problem of how the ordinary fermions acquire mass. Many extensions of technicolor have been suggested in the literature to address this issue. Some of the extensions make use of yet another strongly coupled gauge dynamics, others introduce fundamental scalars. It is even possible to marry supersymmetry and technicolor. Many variants of the schemes presented above exist. A nice review of the major models is the one by Hill and Simmons [30]. It is fair to say that at the moment there is not yet a consensus on which is the correct ETC. Although it is beyond the scope of this initial investigation to 
provide a complete working scheme for mass generation we find it instructive to construct the simplest model able to provide mass to all of the fermions and which does not affect our results, but rather improves them.

We parametrize our ETC, or better our ignorance about a complete ETC theory, with the (re)introduction of a single Higgs type doublet on the top of the minimal walking theory whose main purpose is to give mass to the ordinary fermions. This simple construction leads to no flavor changing neutral currents and does not upset the agreement with the precision tests which our MWT theory already passes brilliantly. We are able to give mass to all of the fermions and the contribution to the beta functions reads:

$$
\begin{aligned}
b_{3} & =\frac{4}{3} N_{g}-11, \\
b_{2} & =\frac{4}{3}\left(N_{g}+1\right)-\frac{22}{3}+\frac{1}{6}, \\
b_{1} & =\frac{4}{3}\left(N_{g}+1\right)+\frac{1}{10},
\end{aligned}
$$

leading to

$$
B_{\text {theory }}=0.71
$$

a value which, at the one loop level, is even closer to the experimental value of 0.72 than the original MWT theory alone. The unification scale is also slightly higher than in MWT alone and it is of the order of $1.2 \times 10^{13} \mathrm{GeV}$. The ETC construction presented above has already been used many times in the literature [20, 21, 22, 23, 24, 25]. We find the results very encouraging. We wish to add that the need for walking dynamics in the gauge sector is important since it helps reducing the value of the S-parameter which is typically large even before taking into account the problems due to the introduction of an ETC sector.

\section{A NEW EXTENSION OF THE STANDARD MODEL}

We wish to improve on the unification point (before taking into account of possible ETC type corrections) and delay it, energy-wise, to avoid the experimental bounds on the proton decay.

We hence need a minimal modification of our extension of the SM with the following properties: i) it is natural, i.e. it does not reintroduce the hierarchy problem, ii) it does not affect the working technicolor sector, iii) it allows for a straightforward unification with 
a resulting theory which is asymptotically free, iv) it yields a phenomenologically viable proton decay rate and possibly leads also to dark matter candidates.

Point i) forces us to add new fermionic-type matter while ii) can be satisfied by modifying the matter content of the SM per se. A simple thing to do is to explore the case in which we consider adjoint fermionic matter for the strong and weak interactions. We will show that this is sufficient to greatly improve the proton decay problem while also improving unification with respect to the MWT theory. To be more specific, we add one colored Weyl fermion transforming solely according to the adjoint representation of $S U(3)$ and a Weyl fermion transforming according to the adjoint representation of $S U_{L}(2)$. These fermions can be identified with the gluino and wino in supersymmetric extensions of the SM. The big hierarchy is still under control in the present model.

Since our theory is not supersymmetric the introduced fermions need not be degenerate with the associated gauge bosons. Their masses can be of the order of, or larger than, the electroweak scale. Finally, naturality does not forbid the presence of a fermion associated to the hypercharge gauge boson and hence this degree of freedom may occur in the theory. Imagining a unification of the value of the masses at the unification scale also requires the presence of such a $U(1)$ bino-type fermion.

In this case the one-loop beta function coefficients are

$$
\begin{aligned}
b_{3} & =\frac{4}{3} N_{g}-11+2, \\
b_{2} & =\frac{4}{3}\left(N_{g}+1\right)-\frac{22}{3}+\frac{4}{3}, \\
b_{1} & =\frac{4}{3}\left(N_{g}+1\right) .
\end{aligned}
$$

This gives $B_{\text {theory }}=13 / 18 \sim 0.72(2)$ which is in excellent agreement with the experimental value. Note also that the unification scale is $M_{G U T} \sim 2.65 \times 10^{15} \mathrm{GeV}$ which brings the proton decay within the correct order of magnitude set by experiments.

\section{A. Unifying Technicolor as Well}

We can make the technicolor coupling unify with the SM couplings, as done in the MWT section. Using Eq. (17), we find now $X \sim 10^{8} \mathrm{GeV}$. We recall here that $X$ is the scale above which our technicolor theory becomes an $S U(3)$ gauge theory with the fermions transforming according to the fundamental representation. 


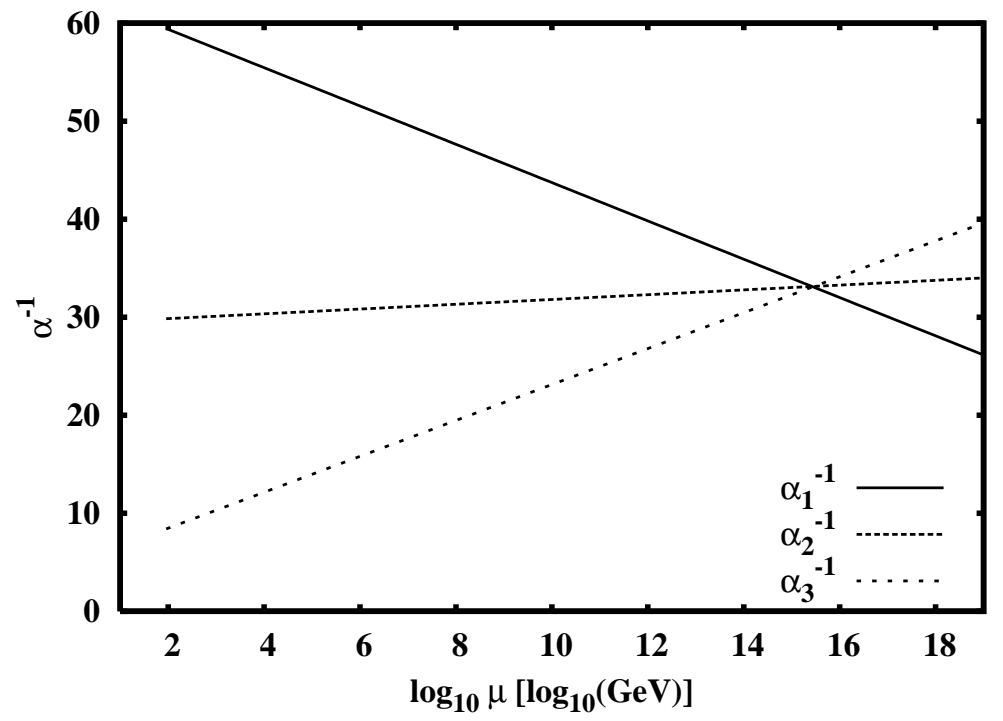

FIG. 3: The running of the three SM gauge couplings in the new model with also adjoint fermionic matter for the SM gauge groups.
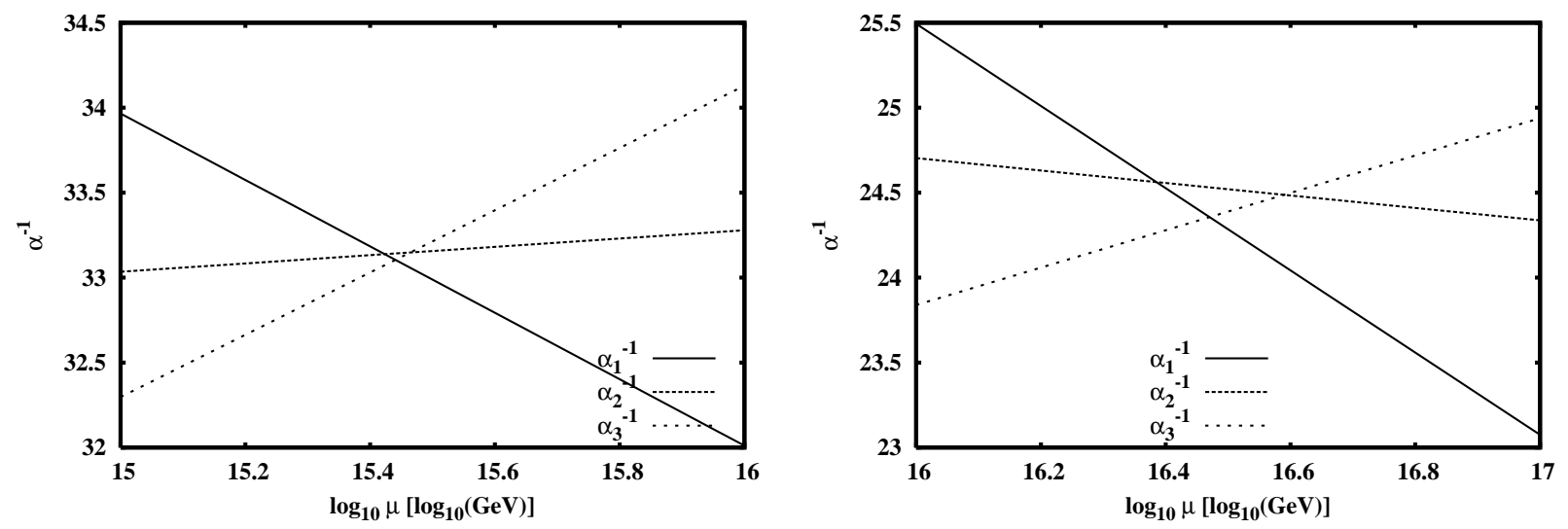

FIG. 4: Left Panel: A zoom around the unification point of the running of the three SM gauge couplings in the new model with extra fermionic adjoint matter for the SM gauge groups. Right Panel: A zoom around the unification point for the couplings in the MSSM.

It is phenomenologically appealing that the scale $X$ is much higher than the electroweak scale. This allows our technicolor coupling to walk for a sufficiently large range of energy to allow for the introduction of extended technicolor interactions needed to give masses to the SM particles. 


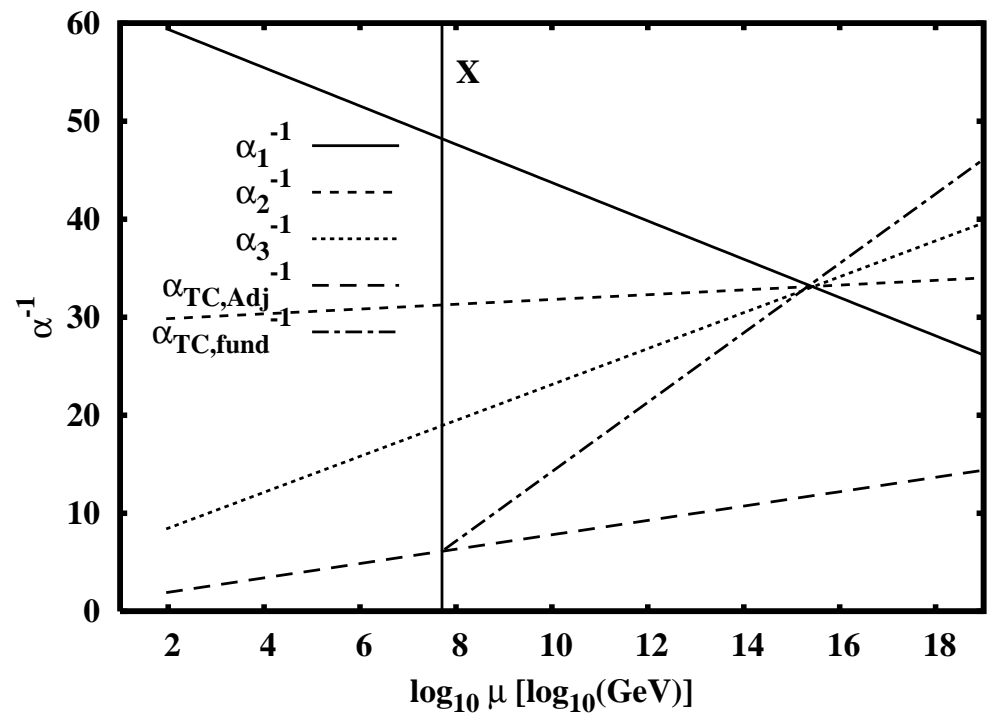

FIG. 5: The running of the three SM gauge couplings as well as the technicolor one. MWT is made to unify with the other three couplings by enhancing the gauge group from $S U(2)$ of technicolor to $S U(3)$ while keeping the same fermionic matter content. We see that the scale where this enhancement of the gauge group should dynamically occur to obtain complete unification is around $10^{8} \mathrm{GeV}$.

\section{B. Comparing with the MSSM and Hint of Dark Matter}

Unification of the SM gauge couplings is considered one of the strongest points in favor of a supersymmetric extension of the SM and hence it is reasonable to compare our results with the SUSY ones. In SUSY, one finds $B_{\text {theory }}=0.714$ which is remarkably close to the experimental value $B_{\exp } \sim 0.72$ but it is not better than the value predicted in the present model which is $0.72(2)$. Obviously this comparison must be taken with a grain of salt since we still need to provide mass to the SM fermions and take care of the threshold corrections. For example according to the model introduced in section III, subsection G, to give masses to all of the fermions yields a theoretical value for the unification which is around 0.76.

There are three possible candidates for dark matter here, depending on which one is the lightest one and on the extended technicolor interactions which we have not yet specified but that we will explore in the future: The chargeless fermion in the adjoint representation of $S U(2)_{L}$, i.e. the wino-like object as well as the bino-type one. The third possibility is the heavy neutrino-like fermion whose dark matter potential features are being currently 
investigated [38].

\section{CONCLUSIONS}

We have introduced a technicolor model which leads to the unification of the SM gauge couplings. At the one-loop level the model provides a higher degree of unification when compared to other technicolor models and to the minimal supersymmetric extension of the SM.

The phenomenology, both for collider experiments and cosmology, of the present extension of the SM is very rich and needs to be explored in much detail.

The model has many features in common with split and non-split supersymmetry [31, 35] and also with very recent models proposed in [36, 37] while others in common with technicolor.

\section{Acknowledgments}

We thank T. Appelquist, B. Bajc, D.D. Dietrich, R. Foadi, M.T. Frandsen, C. Kouvaris and K. Tuominen for discussions or careful reading of the manuscript.

The work of F.S. is supported by the Marie Curie Excellence Grant under contract MEXTCT-2004-013510. F.S. is also supported as Skou fellow of the Danish Research Agency.

[1] S. Weinberg, "Implications Of Dynamical Symmetry Breaking: An Addendum," Phys. Rev. D 19, 1277 (1979); L. Susskind, "Dynamics Of Spontaneous Symmetry Breaking In The Weinberg-Salam Theory," Phys. Rev. D 20, 2619 (1979).

[2] F. Sannino and K. Tuominen, "Orientifold Theory Dynamics and Symmetry Breaking," Phys. Rev. D 71, 051901 (2005). arXiv:hep-ph/0405209.

[3] D. K. Hong, S. D. H. Hsu and F. Sannino, "Composite Higgs from higher representations," Phys. Lett. B 597, 89 (2004) arXiv:hep-ph/0406200.

[4] D. D. Dietrich, F. Sannino and K. Tuominen, "Light composite Higgs and precision electroweak measurements on the Z resonance: An update," Phys. Rev. D 73, 037701 (2006) arXiv:hep-ph/0510217. 
[5] D. D. Dietrich, F. Sannino and K. Tuominen, "Light composite Higgs from higher representations versus electroweak precision measurements: Predictions for LHC," Phys. Rev. D 72, 055001 (2005) arXiv:hep-ph/0505059.

[6] N. Evans and F. Sannino, "Minimal walking technicolour, the top mass and precision electroweak measurements," arXiv:hep-ph/0512080.

[7] S. B. Gudnason, C. Kouvaris and F. Sannino, "Dark matter from new technicolor theories," Phys. Rev. D 74 (2006) 095008 arXiv:hep-ph/0608055]. S. B. Gudnason, C. Kouvaris and F. Sannino, "Towards working technicolor: Effective theories and dark matter," Phys. Rev. D 73, 115003 (2006) arXiv:hep-ph/0603014.

[8] B. Holdom, "Raising The Sideways Scale," Phys. Rev. D 24, 1441 (1981).

[9] K. Yamawaki, M. Bando and K. i. Matumoto, "Scale Invariant Technicolor Model And A Technidilaton," Phys. Rev. Lett. 56, 1335 (1986).

[10] T. W. Appelquist, D. Karabali and L. C. R. Wijewardhana, "Chiral Hierarchies And The Flavor Changing Neutral Current Problem In Technicolor," Phys. Rev. Lett. 57, 957 (1986).

[11] V. A. Miransky and K. Yamawaki, "Conformal phase transition in gauge theories," Phys. Rev. D 55, 5051 (1997) [Erratum-ibid. D 56, 3768 (1997)] arXiv:hep-th/9611142].

V. A. Miransky, T. Nonoyama and K. Yamawaki, "On The Phase Diagram Of Asymptotically Free Gauge Theories With Additional Four Fermion Interaction," Mod. Phys. Lett. A 4, 1409 (1989).

[12] K. D. Lane and E. Eichten, "Two Scale Technicolor," Phys. Lett. B 222, 274 (1989). E. Eichten and K. D. Lane, "Dynamical Breaking Of Weak Interaction Symmetries," Phys. Lett. B 90, 125 (1980).

[13] A. G. Cohen and H. Georgi, "Walking Beyond The Rainbow," Nucl. Phys. B 314, 7 (1989).

[14] M. E. Peskin and T. Takeuchi, "A New Constraint On A Strongly Interacting Higgs Sector," Phys. Rev. Lett. 65, 964 (1990).

[15] T. Appelquist and F. Sannino, "The physical spectrum of conformal SU(N) gauge theories," Phys. Rev. D 59, 067702 (1999) arXiv:hep-ph/9806409.

[16] R. Sundrum and S. D. H. Hsu, "Walking technicolor and electroweak radiative corrections," Nucl. Phys. B 391, 127 (1993) arXiv:hep-ph/9206225].

[17] T. Appelquist, P. S. Rodrigues da Silva and F. Sannino, "Enhanced global symmetries and the chiral phase transition," Phys. Rev. D 60, 116007 (1999) arXiv:hep-ph/9906555. Z. y. Duan, 
P. S. Rodrigues da Silva and F. Sannino, "Enhanced global symmetry constraints on epsilon terms," Nucl. Phys. B 592, 371 (2001) arXiv:hep-ph/0001303.

[18] D. D. Dietrich and F. Sannino, "Conformal window of SU(N) gauge theories with fermions in higher dimensional representations". Phys. Rev. D 75, 085018 (2007) arXiv:hep-ph/0611341.

[19] E. Witten, "An SU(2) Anomaly," Phys. Lett. B 117, 324 (1982).

[20] E. H. Simmons, "Phenomenology of a technicolor model with heavy scalar doublet" Nucl. Phys. B 312, 253 (1989).

[21] M. Dine, A. Kagan and S. Samuel, "Naturalness in supersymmetry, or raising the supersymmetry breaking scale," Phys. Lett. B 243, 250 (1990).

[22] A. Kagan and S. Samuel, "The Family mass hierarchy problem in bosonic technicolor," Phys. Lett. B 252, 605 (1990).

[23] A. Kagan and S. Samuel, "Renormalization group aspects of bosonic technicolor," Phys. Lett. B 270, 37 (1991).

[24] C. D. Carone and E. H. Simmons, "Oblique corrections in technicolor with a scalar," Nucl. Phys. B 397, 591 (1993) arXiv:hep-ph/9207273.

[25] C. D. Carone and H. Georgi, "Technicolor with a massless scalar doublet," Phys. Rev. D 49, 1427 (1994) arXiv:hep-ph/9308205.

[26] W. M. Yao et al. [Particle Data Group], "Review of particle physics," J. Phys. G 33, 1 (2006).

[27] L. F. Li and F. Wu, "Coupling constant unification in extensions of standard model," Int. J. Mod. Phys. A 19, 3217 (2004) arXiv:hep-ph/0304238.

[28] N. D. Christensen and R. Shrock, "On the unification of gauge symmetries in theories with dynamical symmetry breaking," Phys. Rev. D 72, 035013 (2005) arXiv:hep-ph/0506155.

[29] N. D. Christensen and R. Shrock, "Technifermion representations and precision electroweak constraints," Phys. Lett. B 632, 92 (2006) arXiv:hep-ph/0509109.

[30] C. T. Hill and E. H. Simmons, Phys. Rept. 381, 235 (2003) [Erratum-ibid. 390, 553 (2004)] arXiv:hep-ph/0203079.

[31] G. F. Giudice and A. Romanino, "Split supersymmetry," Nucl. Phys. B 699, 65 (2004) [Erratum-ibid. B 706, 65 (2005)] arXiv:hep-ph/0406088].

[32] S. Aoki et al. [JLQCD Collaboration], "Nucleon decay matrix elements from lattice QCD," Phys. Rev. D 62 (2000) 014506 arXiv:hep-lat/9911026.

[33] G. G. Ross, "Grand Unified Theories," Frontiers in Physics, ABP. 
[34] Y. Suzuki et al. [TITAND Working Group], "Multi-Megaton water Cherenkov detector for a proton decay search: TITAND arXiv:hep-ex/0110005.

[35] N. Arkani-Hamed and S. Dimopoulos, "Supersymmetric unification without low energy supersymmetry and signatures for fine-tuning at the LHC," JHEP 0506, 073 (2005) [arXiv:hep-th/0405159].

[36] B. Bajc and G. Senjanovic, "Seesaw at LHC," arXiv:hep-ph/0612029.

[37] I. Dorsner and P. F. Perez, "On fermion masses, unification, proton decay and the UV cutoff of adjoint SU(5)," arXiv:hep-ph/0612216.

[38] Private communication with Kimmo Tuominen.

[39] The adjoint representation of $S U(2)$ - technicolor.

[40] Note that here we are using a different normalization than the one adopted in [18].

[41] Such as the five and the ten dimensional representation of the unifying gauge group $S U(5)$.

[42] Since the technicolor dynamics is strongly coupled at the electroweak scale the last point on the unification of all of the couplings is meant to be only illustrative.

[43] To achieve such as a spontaneous breaking of the gauge group one needs new matter fields around or slightly above the grand unified scale transforming with respect to both the gauge groups. 